\title{
Editorial: Exploring Human-Computer Interactions in Virtual Performance and Learning in the Context of Rehabilitation
}

\author{
Rachel Proffitt ${ }^{1 *}$, Stephanie Glegg ${ }^{2}$, Tal Krasovsky ${ }^{3,4}$, Belinda Lange ${ }^{5}$, Danielle Levac ${ }^{6}$, \\ Anat V. Lubetzky ${ }^{7}$, Wendy A. Powell ${ }^{8}$ and Maxime T. Robert ${ }^{9}$
}

${ }^{1}$ Department of Occupational Therapy, University of Missouri, Columbia, MO, United States, ${ }^{2} B C$ Children's Hospital Research Institute, Vancouver, BC, Canada, ${ }^{3}$ Department of Physical Therapy, University of Haifa, Haifa, Israel, ${ }^{4}$ Pediatric Rehabilitation Department, Edmond \& Lily Safra Children's Hospital, Sheba Medical Center, Ramat-Gan, Israel, ${ }^{5}$ Caring Futures Institute, College of Nursing and Health Sciences, Flinders University, Adelaide, SA, Australia, ${ }^{6}$ School of Rehabilitation, University of Montreal, Montreal, QC, Canada, 'Department of Physical Therapy, New York University, New York, NY, United States, ${ }^{8}$ Department of Cognitive Science and Artificial Intelligence, Tilburg University, Tilburg, Netherlands, ${ }^{9}$ Center for Interdisciplinary Research in Rehabilitation and Social Integration, Department of Rehabilitation, Laval University, Quebec City, QC, Canada

Keywords: virtual reality, human-computation interaction, learning, rehabilatation, editorial

Editorial on the Research Topic

Exploring Human-Computer Interactions in Virtual Performance and Learning in the Context of Rehabilitation

\section{OPEN ACCESS}

Edited and reviewed by: Albert Rizzo,

University of Southern California, United States

*Correspondence: Rachel Proffitt

proffittrm@health.missouri.edu

Specialty section:

This article was submitted to Virtual Reality in Medicine,

a section of the journal

Frontiers in Virtual Reality

Received: 05 August 2021

Accepted: 24 August 2021

Published: 10 September 2021

Citation:

Proffitt $R$, Glegg $S$, Krasovsky $T$,

Lange B, Levac D, Lubetzky AV,

Powell WA and Robert MT (2021)

Editorial: Exploring Human-Computer Interactions in Virtual Performance and

Learning in the Context

of Rehabilitation.

Front. Virtual Real. 2:754020.

doi: 10.3389/frvir.2021.754020
Virtual reality (VR) environments are becoming increasingly prominent in rehabilitation (Howard, 2017), but much remains to be understood about the influence of human factors on the effectiveness of virtual interactions. VR environments relevant to rehabilitation contexts vary in terms of interaction interface requirements and methods of input and control (Lubetzky et al., 2020). Other technological affordances such as level of immersion, the type of feedback provided, or the complexity of audiovisual graphics may also influence outcomes in rehabilitation contexts, and the extent to which interactions in virtual environments transfers to improved outcomes in real life. In order to support the emerging evidencebase, it is important to understand how user characteristics interact with the technological attributes of virtual environments to influence performance, behaviors and learning. The goal of this Research Topic was to highlight insightful and multidisciplinary examples of the potential impact of human-computer interactions in virtual environments in the context of rehabilitation.

Many of the articles in this Research Topic discuss the need for human-computer interfaces to individualize interventions by tapping into the emotive aspects of rehabilitation. To this end, Elor and Kurniawan present a bridging review that links immersive virtual reality to rehabilitation. They propose a theoretical framework that informs design of a computationally-adept medium for physical rehabilitation. Their framework expands the perception of VR by proposing a rehabilitation-specific VR system which would integrate emotional feedback, among other outcomes collected in real-time.

Croucher et al. present the use of embedded context-sensitive attractors to guide attention and walking direction during virtual reality-based clinical walking interventions. The authors posit that verbal directions provided in virtual environments reduce participant autonomy and they argue that the investigation of such context-sensitive attractors is a step towards enhanced virtual rehabilitation. Willis et al. explored the spatial relationships between visual and tactile stimuli. In their investigation 
of visual capture of a tactile sensation, the authors promote the concept that perception is individual and matters to the design of virtual environments for rehabilitation, specifically for individuals with phantom limb pain.

Two other studies in this Research Topic present further findings to support the need for personalization in virtual reality environments. De Castro et al. explore commercially available accelerometers for home practice of motor tasks in persons post-stroke. The findings from this study highlight the need for machine learning algorithms to present clinically-relevant data and guide rehabilitation treatment planning. Brown and Powell present evidence to support the need for a pre-exposure baseline measure of cybersickness in people with chronic pain to support better interpretation of findings from research studies using head-mounted displays in rehabilitation. Each of these articles provide robust evidence for the need to personalize components of human-computer interactions in virtual environments, as well as to better the understanding of specific patient populations, to support optimal rehabilitation and learning.

The remaining articles in this Research Topic discuss transfer or generalization of performance in a virtual environment to the real world. Joyner et al. compared performance of a dexterous task in VR and real-world environments in order to improve VRbased training for individuals with technologically-advanced upper limb prostheses. The authors suggest that unrealistic physics and object occlusion are important characteristics of the virtual environment to consider when designing for success in the real world. Keshner and Lamontagne argue that characteristics of virtual environments must be executed and applied carefully to support transfer to real-world performance. Though their research is in balance and gait in neurological

\section{REFERENCES}

Howard, M. C. (2017). A Meta-Analysis and Systematic Literature Review of Virtual Reality Rehabilitation Programs. Comput. Hum. Behav. 70, 317-327. doi:10.1016/j.chb.2017.01.013

Lubetzky, A. V., Kelly, J., Wang, Z., Gospodarek, M., Fu, G., Sutera, J., et al. (2020). Contextual Sensory Integration Training via Head Mounted Display for Individuals with Vestibular Disorders: a Feasibility Study. Disabil. Rehabil. Assistive Tech. 15, 1-11. doi:10.1080/17483107.2020.1765419

Conflict of Interest: The authors declare that the research was conducted in the absence of any commercial or financial relationships that could be construed as a potential conflict of interest. disorders, they highlight how virtual environments that enhance perception-action coupling lead to better real-world performance. Harvie discusses specific features of VR that can support active learning, improve access, and increase transfer of learning and skills to the real world for individuals with chronic diseases taking part in self-management interventions. Lastly, Furmanek et al. present research on how components of the virtual environment, specifically hand-object collisions, impact perception-action dynamics and reach-to-grasp coordination both in the virtual environment and in the real world. Together, these studies add to the literature on virtual environment design to advance our understanding of its importance on transfer of training.

Hoeg and colleagues present a systematic review and metaanalysis of virtual reality-based rehabilitation in older adults. The review and analyses classify studies based on level of immersion and present a taxonomy of virtual rehabilitation systems. The paper is a call to action to the field of virtual rehabilitation to address the ambiguity of definition of VR and immersion. All the papers in this Research Topic illuminate what is currently known about the influence of human factors in learning in virtual rehabilitation contexts, identify subsequent research directions, and inform decision-making about clinical use of VR environments in rehabilitation.

\section{AUTHOR CONTRIBUTIONS}

RP drafted and edited the article. SG, TK, BL, DL, AL, WP, and MR edited the article. All authors approved the final version for submission.

Publisher's Note: All claims expressed in this article are solely those of the authors and do not necessarily represent those of their affiliated organizations, or those of the publisher, the editors and the reviewers. Any product that may be evaluated in this article, or claim that may be made by its manufacturer, is not guaranteed or endorsed by the publisher.

Copyright (c) 2021 Proffitt, Glegg, Krasovsky, Lange, Levac, Lubetzky, Powell and Robert. This is an open-access article distributed under the terms of the Creative Commons Attribution License (CC BY). The use, distribution or reproduction in other forums is permitted, provided the original author(s) and the copyright owner(s) are credited and that the original publication in this journal is cited, in accordance with accepted academic practice. No use, distribution or reproduction is permitted which does not comply with these terms. 